\title{
Regulation of Reprogramming and Cellular Plasticity through Histone Exchange and Histone Variant Incorporation
}

\author{
Xavier Gaume and Maria-Elena Torres-Padilla \\ Institut de Génétique et de Biologie Moléculaire et Cellulaire, CNRS/INSERM U964, U de S, F-67404 \\ Illkirch, CU de Strasbourg, France \\ Correspondence: metp@igbmc.fr
}

\begin{abstract}
Early embryonic cells are totipotent and can generate a complete organism including embryonic and extraembryonic tissues. After division, cells lose their potency as they move toward a pluripotent state characterized by decreased cellular plasticity. During this transition, drastic changes in transcriptional programs occur in parallel with global chromatin reorganization. The epigenetic mechanisms governing the changes in chromatin signatures during the transitions of cellular plasticity states are starting to be understood. Among these mechanisms, recent studies highlight the importance of histone variant incorporation and/or eviction from chromatin in the regulation of the chromatin state that is linked to cellular potential. In this review, we discuss the role of histone variants during in vivo and in vitro reprogramming events. These results sustain the hypothesis that histone variants and histone exchange are key actors in the establishment of cellular plasticity programs.
\end{abstract}

Development starts with the fusion of two highly differentiated gametes (the spermatocyte and the oocyte) to form the zygote. The zygote is a totipotent cell that gives rise to all the embryonic and extraembryonic lineages. Generating a complete functional organism requires a precise order of molecular, cellular, and tissue-level events that combine cell division, reorganization, migration, differentiation, and cell death. These changes are driven - or at least supported - by a plethora of drastic spatiotemporal changes in transcriptional programs occurring in embryonic cells despite containing similar genetic information. Thus, epigenetic regulation plays a critical role in the control of transcriptional programs during development.

Epigenetic regulation includes DNA methylation, histone posttranslational modifications, chromatin remodeling, noncoding RNA, and histone variants. All of these elements can alter the properties of the chromatin and its interacting partners, thus controlling transcription. Among these epigenetic factors, histone variants are emerging as key regulators of transcription in embryonic stem (ES) cells and during development. Here, we put forward a hypothesis to consider histone replacement and chromatin assembly as a main driver of the chromatin configuration that sustains totipotency. We will first build on the known characteristics and mechanisms of histone variants with respect to their canonical counterparts in an exhaustive review and will subsequently elaborate our hypothesis that global histone exchange serves to consolidate totipotency in vivo and in vitro.

\section{HISTONE VARIANTS: FACTS AND PROPERTIES}

Canonical histones are encoded by several genes organized in clustered repeat arrays. They are transcribed dur- ing $\mathrm{S}$ phase and their incorporation onto DNA only occurs during DNA synthesis. Histone variants differ from their canonical counterpart in amino acid sequence. In contrast to canonical histones, histone variants are typically encoded by a single copy gene whose transcription can occur outside of $\mathrm{S}$ phase leading to deposition of the variant independently of replication. Histone variant deposition onto chromatin is tightly regulated by specific histone chaperones and is thought to occur at specialized domains, although the mechanisms for specific targeting are unknown. In this section, we will introduce $\mathrm{H} 2 \mathrm{~A}$ and $\mathrm{H} 3$ histone variants and we will provide a brief description of their expression patterns during mouse preimplantation development.

\section{H3 Variants}

Mammalian cells possess two canonical H3 histones, H3.1 and H3.2, that differ only in a single residue (Cys to Ser substitution at position 96) (Hake and Allis 2006). Mammals also have additional $\mathrm{H} 3$ variants: $\mathrm{H} 3.3$, the H3 testis-specific variants H3.4 (previously referenced as H3t) (Witt et al. 1996) and H3.5 (Schenk et al. 2011), the centromeric variant cenH3 (also known as CENP-A), and the primate-specific variants H3.Y.1 and H3.Y.2 (Wiedemann et al. 2010). Mammalian H3.3 is encoded by two intron-containing genes (h3f3a and h3f3b) encoding identical proteins (Wellman et al. 1987) that differ from the canonical $\mathrm{H} 3.1$ and $\mathrm{H} 3.2$ by only four or five amino acids, respectively, including residue 31 in the amino-terminal tail (Ala in $\mathrm{H} 3.1 / \mathrm{H} 3.2$ vs. Ser in H3.3). H3.3 incorporation into chromatin is performed by the chromatin remodeling complexes Chd1 (Konev et al. 2007) and ATRX/Daxx (Drané et al. 2010; Lewis et al. 2010) and the histone chaperone HIRA 
(Tagami et al. 2004). H3.3 is associated with transcriptionally active regions (Schwartz and Ahmad 2005; Jin et al. 2009; Goldberg et al. 2010; Chen et al. 2013), and H3.3-containing nucleosomes are intrinsically less stable in vitro, especially when associated with the $\mathrm{H} 2 \mathrm{~A}$ variant H2A.Z (Jin and Felsenfeld 2007). In the mouse oocyte, $\mathrm{H} 3.3$ is present as a maternal factor and is incorporated before H3.1/H3.2 into the male pronucleus after fertilization (Torres-Padilla et al. 2006). The double H3.3 knockout (h3f3a and h3f3b) results in embryonic lethality in the mouse (Jang et al. 2015), but not in Drosophila, where deletion of H3.3 leads to sterility (Hödl and Basler 2009; Sakai et al. 2009).

\section{H2A Variants: H2A.X, H2A.Z, macroH2A, and H2A.B}

H2A has the largest number of variants described, with biochemical and functional properties that clearly distinguish each of them.

H2A.X possesses a carboxy-terminal tail containing a serine-glutamine (SQ)-rich motif and its function during DNA repair has been studied extensively. After a DNA double-strand break, H2A.X is phosphorylated by the ATM, ATR, and DNA-PK kinases on S139 ( $\gamma$ H2A.X) (Rogakou et al. 1998) leading to the recruitment of several DNA repair proteins (Fernandez-Capetillo et al. 2004). Additional biological functions of H2A.X, nonrelated to the DNA damage response, have been described (for review, see Turinetto and Giachino 2015). In mice, after fertilization, H2A.X is detected by immunofluorescence in both pronuclei and remains present at the two- and four-cell stages; subsequently the intensity of the signal decreases from the morula stage (ZieglerBirling et al. 2009; Nashun et al. 2010; Wossidlo et al. 2010). H2A. $X^{-/-}$mice are viable but display genome instability, a growth delay, and male infertility (Celeste et al. 2002).

$H 2 A . Z$ is $60 \%$ identical to canonical $\mathrm{H} 2 \mathrm{~A}$ and is wellconserved during evolution, suggesting an important role in the organism. Vertebrates possess two H2A.Z genes (H2A.Z.1 and H2A.Z.2) that encode proteins that differ by three residues (Eirín-López et al. 2009). In yeast, the incorporation of H2A.Z (Htz1) into chromatin is mediated by the histone chaperone Chzl (Luk et al. 2007) and the histone exchanging nucleosome remodeling complex SWR1 (Mizuguchi et al. 2004). Although no H2A.Z-specific factors involved in H2A.Z incorporation have been identified in metazoans, a recent article identified ANP32E displaying histone chaperone activity specific for the H2A.Z/H2B dimer eviction from chromatin (Mao et al. 2014; Obri et al. 2014). In mice, embryos lacking H2A.Z fail to develop beyond the blastocyst stage (Faast et al. 2001). By immunofluorescence, H2A.Z signal is lost shortly after fertilization and reappears at the late two-cell stage or late morula stage depending on the studies (Nashun et al. 2010; Bošković et al. 2012). At the blastocyst stage, H2A.Z is detected at higher level in the nuclei of trophectoderm cells compared with inner cell mass cells (Rangasamy et al. 2003; Nashun et al. 2010).
MacroH2A is a vertebrate-specific histone $\mathrm{H} 2 \mathrm{~A}$ variant. MacroH2A variants are atypical histone variants because of the presence of a large carboxy-terminal nonhistone domain called the macro domain $(>210$ amino acids) (Pehrson and Fried 1992). Mammalian cells encode three macroH2A proteins: macroH2A.2 and the two spliced variants macroH2A.1.1 and macroH2A.1.2. $\mathrm{MacroH} 2 \mathrm{~A}$ is generally considered as transcriptionally repressive because of its association with silenced chromatin (Costanzi and Pehrson 1998). In vitro, macroH2A has inhibitory effects on transcription factor binding (Angelov et al. 2003) and transcription initiation (Doyen et al. 2006). MacroH2A dynamics have been studied by FRAP (fluorescence recovery after photobleaching), which reveals that the mobility of macroH2A is lower than that of H2A suggesting a more stable association to the chromatin (Gaume et al. 2011). Double knockout mice for macroH2A.1 and macroH2A.2 display impaired prenatal and postnatal growth despite a relatively normal early development (Pehrson et al. 2014). MacroH2A is present in mouse oocytes and then lost in the zygote shortly after fertilization (Chang et al. 2005; Nashun et al. 2010) and becomes detectable again at the morula stage and persists in the blastocyst in both trophectoderm and inner cell mass cells (Chang et al. 2005; Nashun et al. 2010).

H2A.B (previously referenced as $\mathrm{H} 2 \mathrm{Abbd}$ for $\mathrm{H} 2 \mathrm{~A}$ Barr body-deficient) is a divergent $\mathrm{H} 2 \mathrm{~A}$ variant (only $48 \%$ sequence identity with $\mathrm{H} 2 \mathrm{~A}$ ) found exclusively in mammals (Chadwick and Willard 2001). H2A.B is encoded by three copies of the h2afb gene. In vitro studies have demonstrated that $\mathrm{H} 2 \mathrm{~A}$.B confers a lower stability to the nucleosome (Eirín-López et al. 2008) and is more mobile than H2A in cells (as assessed by FRAP) (Gautier et al. 2004) and that H2A.B-containing nucleosomes organize only $118 \mathrm{pb}$ of DNA (Bao et al. 2004). All these points argue for a positive role of H2A.B in chromatin accessibility and transcription (Zhou et al. 2007). In agreement, H2A.B is found associated with actively transcribed genes in HeLa cells (Tolstorukov et al. 2012).

Additional $\mathrm{H} 2 \mathrm{~A}$ and $\mathrm{H} 2 \mathrm{~B}$ variants exist, which are specifically expressed in the germline, in particular in the testis, that include TH2A, TH2B, H2A.L.1, and H2A.L.2, which are thought to be involved in facilitating incorporation of transition proteins and removal of histones necessary for the formation of the mature sperm (Govin et al. 2007; Soboleva et al. 2012; Montellier et al. 2013).

\section{H1 Isoforms}

The linker histone H1 plays critical roles in the compaction of chromatin and the arrangement of higher-order chromatin structure, as well as in the recruitment of numerous proteins that modify chromatin fiber accessibility or conformation (McBryant et al. 2010). Eleven different $\mathrm{H} 1$ variants have been identified in mammals so far, including somatic (H1.0 to H1.5 and H1.10) or germ cell-specific variants (testis-specific H1.6 [previously referenced as H1t], H1.7, oocyte-specific H1.8 
[previously referenced as H1oo]) and H1.9 (for review, see Happel and Doenecke 2009). Although none of the H1 isoforms are essential for mouse development, presumably because of compensation (Sirotkin et al. 1995; Lin et al. 2000; Rabini et al. 2000; Fan et al. 2001), they are important for the fine regulation of gene expression (Alami et al. 2003; Happel and Doenecke 2009), acting both as activators and repressors of transcription (Clausell et al. 2009; Kamieniarz et al. 2012).

Because of their specific features and diversity, histone variants confer additional regulatory levels to tightly control gene expression, DNA repair, and chromosome compaction and recombination. In this review, we discuss recent findings involving histone variants in the regulation of transcription during mammalian development. Most of the work presented below concerns the mouse embryo as a model system.

\section{HETEROCHROMATIN ESTABLISHMENT AND MAINTENANCE BY HISTONE VARIANTS: REGULATION OF REPETITIVE ELEMENTS}

After fertilization, genome-wide epigenetic reprogramming includes de novo establishment of chromatin domains such as heterochromatin. Heterochromatin is a condensed chromatin state resulting in transcriptional silencing of the corresponding genomic regions. Heterochromatin can be classified into constitutive versus facultative. Contrary to facultative heterochromatin, constitutive heterochromatin corresponds to chromosomal regions that are never converted into euchromatin, such as centromeres, telomeres, and repetitive elements. An example of facultative heterochromatin is the inactive $\mathrm{X}$ chromosome in female mammals that can be either packaged into heterochromatin and silenced or organized into euchromatin and expressed. At the molecular level, heterochromatin is characterized by specific epigenetic marks including DNA methylation, repressive histone posttranslational modifications, the presence of characteristic heterochromatin proteins, and the incorporation of specific histone variants in the nucleosome core particle.

Eukaryotic genomes contain a large proportion of repetitive sequences organized in clusters (rDNA, centromeres, and telomeres) or interspersed in the genome (transposable elements). Repetitive elements constitute a permanent threat for genomic stability. Thus, heterochromatinization of these loci is crucial to silence them and prevent, for example, recombination or retrotransposition. The specific presence of histone variants on repetitive sequences suggests an important role in the formation and/or maintenance of heterochromatin on these sequences.

Genome-wide localization studies of H3.3 have demonstrated that in addition to its association with active histone modifications, $\mathrm{H} 3.3$ is also enriched in $(\text { TTAGGG })_{n}$ repeats, hallmark of vertebrate telomeres (Goldberg et al. 2010). H3.3 localization at telomeres was confirmed by immunofluorescence in mouse ES cells (Wong et al. 2009). H3.3 is incorporated into telo- meres during $\mathrm{S}$ phase, and a phosphorylated form of $\mathrm{H} 3.3$ (H3.3S31P) is detected on telomeres during mitosis (Wong et al. 2009). Interestingly, upon differentiation, the telomeric signal of H3.3S31P decreases, concomitantly with an increased association of heterochromatin marks (H3K9me3 and H4K20me3) at telomeres (Wong et al. 2009). The H3.3 histone chaperone ATRX colocalizes with H3.3 on telomeres (Wong et al. 2010) and is responsible for H3.3 deposition at these regions (Goldberg et al. 2010; Lewis et al. 2010). To support these observations, chromatin immunoprecipitation (ChIP) analysis has also shown that ATRX is physically associated with telomeres (Goldberg et al. 2010). Interestingly, ATRX depletion leads to the up-regulation of the telomeric repeat containing RNA (TERRA) suggesting that H3.3 is important for proper silencing of telomeric repeats (Goldberg et al. 2010), at least partially by recruiting HP1 $\alpha$ (Wong et al. 2010).

H3.3 has also been associated with endogenous retroviral elements (Classes I and II endogenous retroviruses [ERVs]) (Elsässer et al. 2015). As is the case on telomeres, H3.3 deposition on ERVs is performed by ATRX/Daxx and not by HIRA (Elsässer et al. 2015). In this study, the authors demonstrated that ATRX/ Daxx-dependent deposition of H3.3 on ERVs is important for their repression through the recruitment of the histone methyltransferase ESET, which in turn establishes H3K9me3 on ERVs (Elsässer et al. 2015).

H3.3, together with ATRX/Daxx, is also found on pericentric DNA repeats in mouse embryo fibroblasts (MEFs) (Drané et al. 2010). The absence of any of these partners leads to a decrease in pericentric transcripts (Drané et al. 2010). Similarly, in mouse embryos, H3.3 localizes to the paternal pericentric chromatin during the first S phase and is important for pericentric heterochromatin establishment and integrity (Santenard et al. 2010). In this context, the authors propose the following model: H3.3 promotes the transcription of major satellite RNA, which in turn leads to an efficient silencing of pericentromeric domains (Santenard et al. 2010). In neurons, a recent study has shown that ATRX is specifically recruited to centromeric repeats by the combinatorial histone modifications (H3K9me3 and H3K9S10Ph) (Noh et al. 2015) suggesting a link between specific histone modifications and histone variant deposition.

Although the role of H3.3 in the transcription and the establishment of heterochromatin on repetitive elements (ERVs, telomeres, pericentromeres) in not fully understood (Fig. 1), H3.3-dependent establishment of heterochromatin is important for the maintenance of genome integrity of cells in culture (Jang et al. 2015). Heterochromatin establishment may involve unknown additional factors depending on the genetic environment or the cellular context. It is tempting to assume that H3.3, in agreement with its association with open chromatin structure and transcription, plays a role in the first step of the establishment of heterochromatin by promoting the transcription of small noncoding RNA (Santenard et al. 2010). When established, heterochromatin is then stabilized by the recruitment of additional factors such as heterochromatin 


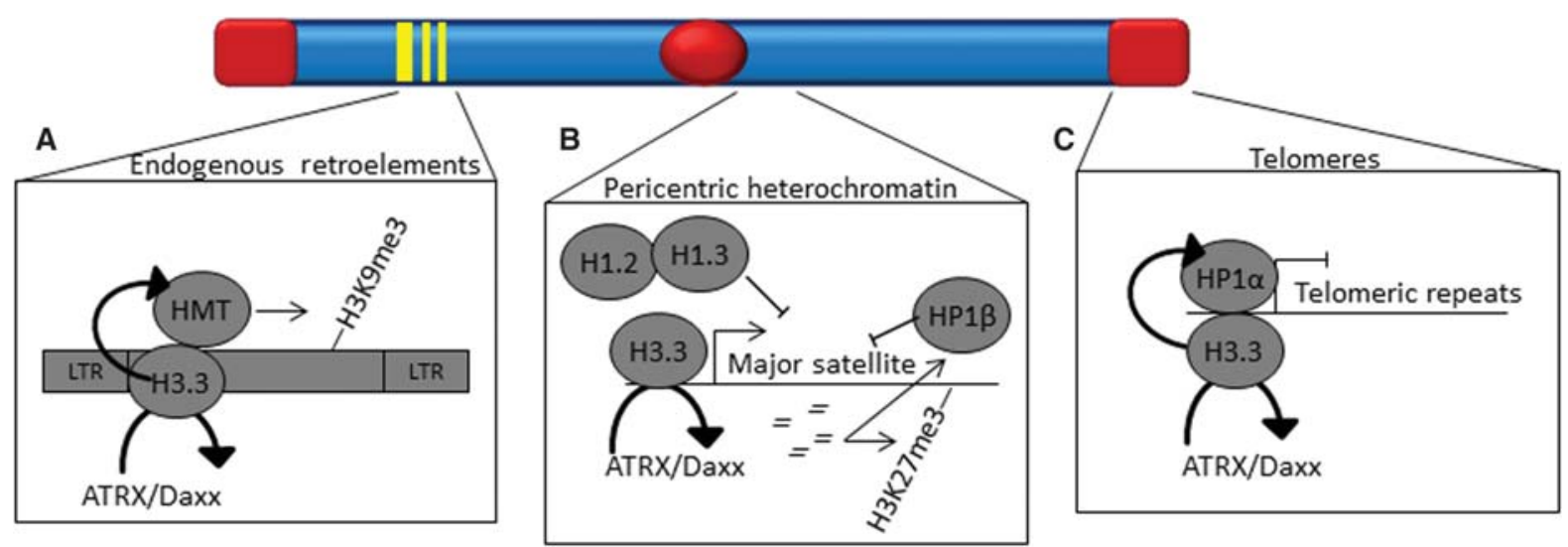

Figure 1. H3.3 is required to establish and maintain heterochromatin on repetitive elements. $(A) \mathrm{H} 3.3$ is associated with endogenous retroviral elements. ATRX/Daxx-dependent deposition of H3.3 on ERVs is important for their silencing through the recruitment of the histone methyltransferase ESET that establishes H3K9me3. (B) H3.3 and ATRX/Daxx are found on pericentric DNA repeats. They are involved in the transcription of major satellite dsRNA, which are then required to recruit HP1 $\beta$ and to establish heterochromatin. $(C)$ ATRX/Daxx deposits H3.3 on telomeric repeats. In this context, H3.3 is important for their silencing through the recruitment of HP1 $\alpha$.

proteins 1 (HP1) (Santenard et al. 2010; Wong et al. 2010) and histone methyltransferases (Elsässer et al. 2015).

Pericentric heterochromatin also contains H2A.Z (Rangasamy et al. 2003). Although the role of H2A.Z on pericentromeres is unknown, the specific interaction of HP1 $\alpha$ with H2A.Z-containing nucleosomes (Fan et al. 2004) suggests that H2A.Z might enhance the recruitment of factors involved in heterochromatin formation and maintenance including HP $1 \alpha$. During spermiogenesis, testis-specific histone $\mathrm{H} 2 \mathrm{~A}$ variants are expressed. Among them, H2A.L.1 and H2A.L.2 are specifically incorporated in pericentric regions (Govin et al. 2007). Although their function is also still unknown, they might guide epigenetic reprogramming of paternal pericentric heterochromatin after fertilization (Govin et al. 2007).

Apart from the repetitive elements of the genome, during developmental progression there are at least two additional paradigms of heterochromatin formation and silencing: $\mathrm{X}$ inactivation and meiotic sex chromosome inactivation (MSCI). Although the former occurs after fertilization, and prior to implantation, the latter takes place during male germline formation. We will not treat these two models in this review and will just mention that histone variants have also been implicated in silencing during MSCI, as is the case of H3.3 (van der Heijden et al. 2007), and in $X$ chromosome inactivation, as is the case of macroH2A (Pasque et al. 2011; Minajigi et al. 2015).

Heterochromatin establishment and maintenance is a complex process that is under constant investigation. Specific histone variants are present in heterochromatin, but their role in the establishment or maintenance of heterochromatin is still unclear. Their presence at these regions may act at multiple levels: (1) by altering the properties of the nucleosomal core particle, making the chromatin less accessible for transcription factors, (2) by allowing transcription of specific RNA potentially involved in heterochromatin formation, and (3) by recruiting specific effectors of heterochromatin formation. Most importantly, their involvement in key developmental epigenetic phenomena makes them a candidate to regulate genome reprogramming and cellular plasticity.

\section{EPIGENETIC REPROGRAMMING: RESETTING CELLULAR PLASTICITY}

Reprogramming is the reversal of an epigenetic state resulting in changes in cellular identity. In mammals, the early embryo undergoes a genome-wide reprogramming event that parallels, and most likely underlies, the acquisition of totipotency. Large-scale reprogramming of differentiated cells can also be obtained in vitro by at least two approaches, somatic cell nuclear transfer (SCNT) and induced pluripotent stem cells (iPSCs). SCNT reprograms to totipotency, whereas iPSC formation entails reprogramming toward pluripotency, both thus resulting in the acquisition of increased cellular plasticity.

\section{Developmental Reprogramming after Fertilization}

The oocyte and the sperm cells are two highly specialized cells. Upon fertilization, they undergo a drastic reprogramming sequence to produce the totipotent zygote. The two parental genomes remain physically segregated $24 \mathrm{~h}$ after fertilization and exhibit different chromatin organizations. The first reprogramming event of the male pronucleus requires the replacement of sperm protamines with maternal histones. Indeed, although some histones remain at specific loci in the sperm nucleus (Hammoud et al. 2009; Brykczynska et al. 2010), most of the paternal genome is unpacked and new histones are incorporated. This exchange occurs rapidly, long before the first mitosis and zygotic genome activation (ZGA). Newly incorporated histones are hyperacetylated and hypomethylated in agreement with the open chromatin structure observed specifically in the paternal pronucleus (for review, see Burton and Torres-Padilla 2014). Histone variants are also incorporated into the male pronucleus 
during this reprogramming event (Fig. 2A) leading to a remarkable asymmetry between the male and female pronuclei. In the mouse oocyte, gamete-specific histone variants are expressed and are subsequently incorporated into the male pronucleus upon fertilization, including H1.8, TH2A, and TH2B. H1.8 expression starts in the oocyte and ends at the two-cell stage, coincident with major ZGA (Tanaka et al. 2001). H1.8 is quickly incorporated into paternal pronucleus after fertilization (Gao et al. 2004). When ectopically expressed in ES cells, H1.8 selectively binds hypomethylated loci and promotes their decondensation (Hayakawa et al. 2012) suggesting that specific incorporation of H1.8 into the male pronucleus may lead to the establishment of an open chromatin structure.

TH2A and TH2B were originally identified as testisspecific $\mathrm{H} 2 \mathrm{~A}$ and $\mathrm{H} 2 \mathrm{~B}$ histone variants, respectively (Shires et al. 1976; Trostle-weige et al. 1982). They are encoded by unique genes (Hist1h2aa and Histh2ba) localized at the end of a large histone gene cluster and have 15- and 16-amino-acid differences compared with H2A and $\mathrm{H} 2 \mathrm{~B}$, respectively. $\mathrm{TH} 2 \mathrm{~A}$ and $\mathrm{TH} 2 \mathrm{~B}$ have been recently found to be expressed in oocytes and in the zygote, where they are both incorporated into the paternal pronucleus after fertilization (Shinagawa et al. 2014). When WT male mice are crossed with double Th $2 a$ and $T h 2 b^{-/-}$knockout females, the authors observed delayed embryonic development compared with controls, underscoring the importance of these two maternally inherited histone variants (Shinagawa et al. 2014). Furthermore, a significant reduction of transcription is observed in two-cell-stage embryos, arguing for a role of TH2A and TH2B in ZGA (Shinagawa et al. 2014). The authors demonstrate that $\mathrm{TH} 2 \mathrm{~A}$ and $\mathrm{TH} 2 \mathrm{~B}$ are specifically involved in the activation of the paternal genome. In the absence of maternal $\mathrm{TH} 2 \mathrm{~A}$ and $\mathrm{TH} 2 \mathrm{~B}$, the level of the active mark $\mathrm{H} 3 \mathrm{~K} 4 \mathrm{me} 3$ in the paternal pronucleus is lower whereas the level of repressive marks is higher (H3K27me3 and DNA methylation) (Shinagawa et al. 2014). These differences have functional consequences on the transcription programs of later developmental
A

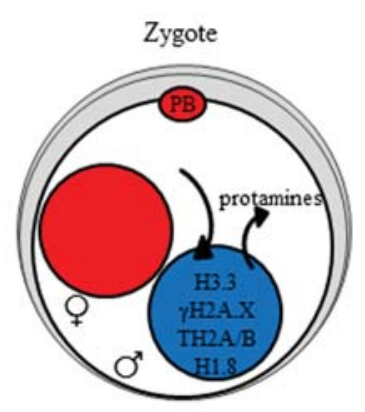

B

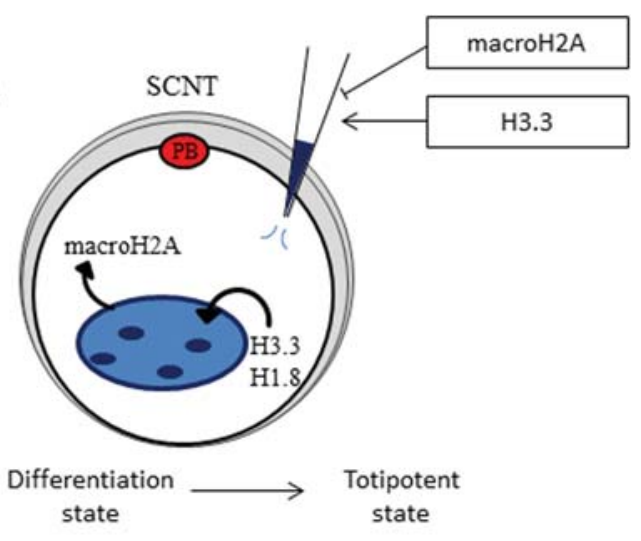

C

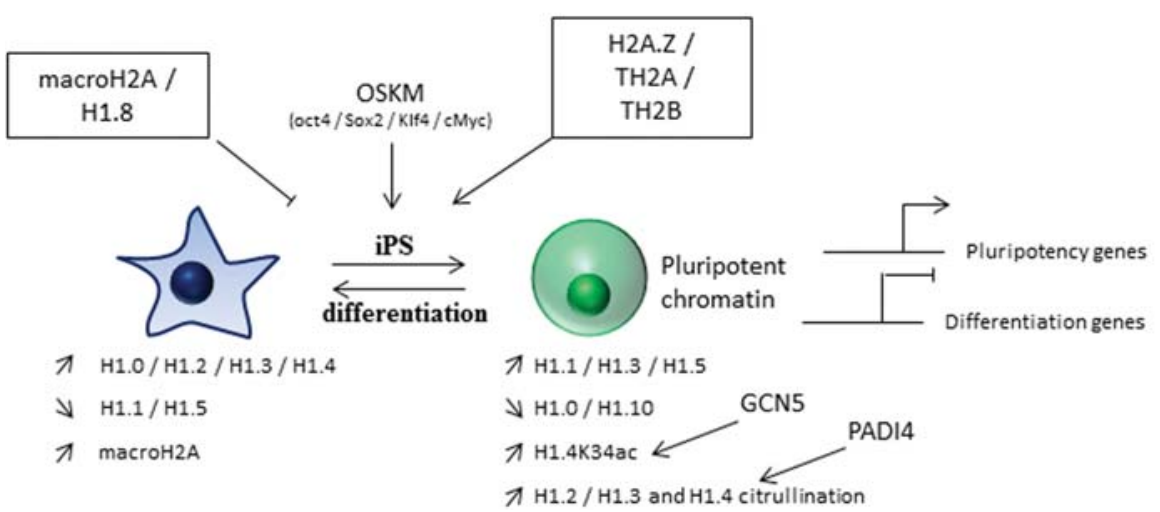

Figure 2. Histone variants control epigenetic reprogramming during cellular plasticity transitions. (A) After fertilization, the H3.3, H1.8, $\gamma$ H2A.X, TH2A, and TH2B histone variants are incorporated into the male pronucleus after protamine removal. They are important to trigger totipotency, to form heterochromatin at specific genomic regions, and to properly regulate ZGA. (B) During somatic cell nuclear transfer (SCNT), a differentiated nucleus acquires a totipotent capacity. H3.3 is important for efficient reprogramming upon SCNT, whereas macroH2A acts as an epigenetic barrier. After implantation in the enucleated oocyte, H3.3 and H1.8 are incorporated in the donor nucleus, whereas macroH2A is quickly removed. $(C)$ During iPS, histone variant composition is dramatically modified to sustain the acquisition of a pluripotent chromatin. H2A.Z, TH2A, and TH2B histone variants favor reprogramming efficiency whereas $\mathrm{H} 1.8$ and macroH2A inhibit it. 
stages, because transcription of Nanog is inhibited from the eight-cell-stage embryo specifically on the paternal allele (Shinagawa et al. 2014). Altogether these observations support a role for the maternal histone variants TH2A and TH2B in the formation of an open chromatin structure on the male genome.

The large genome-wide assembly process taking place soon after fertilization also involves H3.3 because the paternal pronucleus incorporates H3.3 in a replication- and transcription-independent manner, mediated largely by HIRA (van der Heijden et al. 2005; Torres-Padilla et al. 2006; Inoue and Zhang 2014; Lin et al. 2014). The absence of maternal HIRA prevents core histone deposition on the paternal pronucleus and compromises ZGA of ribosomal genes suggesting an additional role of H3.3 in the activation of specific genes from the embryonic genome (Lin et al. 2014). As we have discussed above, H3.3 deposition during early development is also important for the establishment of heterochromatin domains (Santenard et al. 2010). However, it remains unclear whether H3.3 deposition in the paternal pronucleus plays specific roles in facilitating an open chromatin structure or instead the incorporation of $\mathrm{H} 3.3$ represents only an intermediate step prior to the first phase of replication-dependent incorporation of the canonical histone $\mathrm{H} 3.1 / \mathrm{H} 3.2$, which occurs a few hours later.

High levels of $\gamma \mathrm{H} 2 \mathrm{~A} . \mathrm{X}$ are detected in the zygote, with the paternal pronucleus displaying augmented levels compared with the maternal one (Ziegler-Birling et al. 2009). This enrichment in the phosphorylated form of H2A.X seems to be independent of DNA damage as $\gamma \mathrm{H} 2 \mathrm{~A} . \mathrm{X}$ does not colocalize with known DNA damage response proteins (Ziegler-Birling et al. 2009). It is possible that the phosphorylation of H2A.X after fertilization accompanies the DNA demethylation process occurring on the parental chromatin, which has been suggested to occur partially through the base excision repair pathway (Hajkova et al. 2010; Wossidlo et al. 2010).

The zygote is an excellent in vivo model for studying genome-wide reprogramming events using cell biology approaches. During this reprogramming phase, the contribution of histone variants, especially those that are gametespecific, is beginning to be unveiled. Histone variants are involved in the formation of heterochromatic structures and the regulation of ZGA. It is also possible that the global histone exchange from a chromatin structure characterized primarily by incorporation of $\mathrm{H} 3.3$ toward a more "typical" chromatin configuration with the canonical histones H3.1 and H3.2, plays a role in promoting cellular plasticity.

\section{SCNT}

SCNT consists in transplanting a somatic nucleus into an enucleated metaphase II oocyte, leading to the creation a viable embryo. So far, SCNT is the only experimental procedure to reprogram a differentiated nucleus into a totipotent state, thus demonstrating that the potential to regain totipotency is not lost in most adult cells (Gurdon and Melton 2008). Upon introduction into the oocyte, the donor nucleus undergoes a series of reprogramming events controlled by the maternal factors present in the oocyte. This reprogramming toward a totipotent state results in global changes in chromatin composition to reset the epigenetic landscape of the somatic nucleus and leads to a switch in gene expression profile, from a somatic program to an embryonic one. During this process, several maternal factors including histone variants like $\mathrm{H} 3.3$ and H2A.X are incorporated into the donor nucleus (Chang et al. 2010; Nashun et al. 2011; Jullien et al. 2012). However, the oocyte can also fail to reprogram somatic nuclei, suggesting that the donor nucleus contains epigenetic barriers to reprogramming.

Deletion of H3.3 in the mouse oocyte compromises the efficiency of SCNT, preventing the reactivation of a subset of pluripotent genes (Wen et al. 2014). Maternal H3.3 depletion before nuclear transfer results in embryos arresting at the two- or four-cell stage (Wen et al. 2014). The authors show that maternal $\mathrm{H} 3.3$ and not $\mathrm{H} 3.3$ from the donor nucleus is crucial for SCNT and cannot be rescued by H3.2 (Wen et al. 2014). In agreement with this role of H3.3 in SCNT, Jullien et al. (2012) demonstrated that after nuclear transfer in Xenopus, maternal H3.3 is incorporated on major satellite repeats and the regulatory region of Oct4 in an HIRA-dependent manner. Accordingly, H3.3 was also shown to be necessary for reprogramming.

Linker histone variants have also been involved in reprogramming during SCNT. After transplantation, the oocyte $\mathrm{H} 1.8$ is rapidly incorporated in the donor nucleus and persists until the two-cell stage, coincident with ZGA (Gao et al. 2004; Teranishi et al. 2004). In MEFs, H1.8 mobility as assessed by FRAP is higher compared with H1.2 (Teranishi et al. 2004), which would explain the increase in linker histone mobility after SCNT (Jullien et al. 2010). In Xenopus, the linker histone B4 (homologue of H1.8) is involved in reactivation of pluripotencyassociated genes such as Sox2, Nanog, and Oct4 after SCNT (Jullien et al. 2010). Taken together, these data suggest that during SCNT, the linker histone isoforms might be involved in nuclear remodeling and ZGA.

Apart from the roles of $\mathrm{H} 3.3$ and some $\mathrm{H} 1$ isoforms in promoting reprogramming upon SCNT, some studies have addressed the role of other histone variants in SCNT. For instance, macroH2A is quickly removed from the somatic nucleus after transplantation (Chang et al. 2010). The pattern of expression of macroH2A at the protein level is therefore similar to that of naturally produced embryos, as macroH2A is found in the nuclei from the morula stage only, but undetectable at earlier stages (Chang et al. 2005, 2010). Using Xenopus oocytes as an acceptor for a somatic nucleus, Pasque et al. (2011) showed that macroH2A is retained on the inactive $\mathrm{X}$ chromosome of differentiated mouse cells and suggested that this retention renders them resistant to reprogramming. Furthermore, macroH2A depletion in donor MEF nuclei improves reprogramming (Pasque et al. 2011). Altogether, these observations highlight an effect of macroH2A in the resistance to reprogramming during SCNT. Thus, while maternal H3.3 and linker histone variants are important for proper reprogramming upon SCNT, macroH2A interferes with reprogramming (Fig. 2B). 


\section{Reprogramming to Pluripotency upon iPSC Formation}

Differentiated cells can be reprogrammed into a pluripotent state by the introduction of four transcription factors: Oct3 /4, Sox2, Klf4, and cMyc (collectively called OSKM factors) (Takahashi and Yamanaka 2006). The resulting iPSCs provide a remarkable system to understand the molecular mechanisms involved in this change in cell plasticity in vitro (for review, see Apostolou and Hochedlinger 2013). During reprogramming to pluripotency, fibroblasts first down-regulate genes associated with differentiation and subsequently reactivate endogenous pluripotency genes including Oct4, Sox2, and Nanog to sustain a stable pluripotent state and self-renewal. This reprogramming event involves resetting somatic epigenetic marks and the acquisition of a "pluripotent" chromatin structure. However, the acquisition of induced pluripotency is an inefficient process, suggesting the presence of intrinsic epigenetic barriers to reprogramming in the somatic cells. In this context, histone variants are key actors in favoring or inhibiting reprogramming (Fig. 2C).

Citrullination has recently been characterized on histone H1 isoforms H1.2, H1.3, and H1.4 (Christophorou et al. 2014). Citrullination promotes $\mathrm{H} 1$ eviction from chromatin, thus leading to a more open chromatin structure (Christophorou et al. 2014). Interestingly, when the activity of PADI4 - the enzyme responsible for citrullination - is inhibited, the efficiency for iPSC reprogramming is reduced (Christophorou et al. 2014). Thus, it was proposed that during iPSC derivation, citrullination leads to the eviction of $\mathrm{H} 1$ isoforms from the chromatin, leading to the efficient expression of pluripotent genes such as Nanog (Christophorou et al. 2014). On the contrary, overexpression of $\mathrm{H} 1.8$ reduces iPSC generation, suggesting a role of this isoform as a barrier of reprogramming (Shinagawa et al. 2014). During reprogramming of human keratinocytes to $\mathrm{iPSC}$, linker histone variant composition undergoes drastic changes. The expression levels of $\mathrm{H} 1.0$ and $\mathrm{H} 1.10$ decrease during reprogramming, whereas those of H1.1, H1.3, and H1.5 increase (Terme et al. 2011). During iPSC formation, acetylation of H1.4 on K34 increases and is thought to stimulate transcription by increasing H1 mobility (Kamieniarz et al. 2012). These observations suggest a specific role for $\mathrm{H} 1$ isoforms in the changes of chromatin condensation and accessibility during iPSC reprogramming. The lack of specific antibodies for all $\mathrm{H} 1$ isoforms has nevertheless hindered the study of $\mathrm{H} 1$ in these biological processes.

The biological role, if any, of $\gamma \mathrm{H} 2 \mathrm{~A}$.X during iPSC formation remains elusive, although $\gamma \mathrm{H} 2 \mathrm{~A} . \mathrm{X}$ levels are high during the reprogramming process itself (Marion et al. 2009; González et al. 2013). In a recent study, Wu et al. (2014) demonstrate the importance of H2A.X relocalization during iPSC reprogramming for efficient repression of extraembryonic genes and successful tetraploid complementation, suggesting that H2A.X may be an epigenetic mark for the developmental potential of iPSCs. H2A.Z is also important for iPSC formation, as its absence prevents reprogramming of somatic cells, where- as its overexpression increases reprogramming efficiency by OSKM factors (Wang et al. 2015). As the TH2A and TH2B variants play an important role in male nucleus reprogramming after fertilization (see above) the authors also investigated their contribution during iPSC derivation (Shinagawa et al. 2014). The combination of TH2A and $\mathrm{TH} 2 \mathrm{~B}$ overexpression in MEFs enhances iPSC formation ninefold (Shinagawa et al. 2014). Interestingly, coexpression of a phosphomimetic form of their specific chaperone nucleoplasmin (NPM) increases OSKMinduced iPSC generation even further, 18-fold (Shinagawa et al. 2014). TH2A/TH2B and NPM-P overexpression enhance DNA demethylation of the regulatory sequences of Nanog (Shinagawa et al. 2014). Furthermore, TH2A and TH2B specifically associate with the X chromosome during iPSC formation but the effect of these two variants, if any, on the changes in chromatin accessibility of the $\mathrm{X}$ chromosome remains unclear (Shinagawa et al. 2014).

Reprogramming to iPSCs also entails the removal of macroH2A. 1 and macroH2A.2 from the chromatin (Gaspar-Maia et al. 2013). The same remains true in human keratinocytes compared with the corresponding iPSCs (Barrero et al. 2013). Using several reprogramming protocols, Gaspar-Maia et al. (2013) showed that reprogramming is more efficient in double macroH2A knockout fibroblasts compared with WT cells. This effect is independent of the action of macroH2A on the inactive $\mathrm{X}$ chromosome (in contrast to its function in SCNT [Pasque et al. 2011]) as both male and female cells display a similar reprogramming rate (Gaspar-Maia et al. 2013). In human cells, only macroH2A.1 depletion-but not macroH2A.2 depletion-has an enhancing effect on reprogramming into iPSCs (Barrero et al. 2013). Interestingly, macroH2A is associated with $\sim 25 \%$ of the genes that are bound by the OSKM factors (Gaspar-Maia et al. 2013), and its absence promotes the reacquisition of $\mathrm{H} 3 \mathrm{~K} 4 \mathrm{me} 2$ at promoters of pluripotency genes during reprogramming (Barrero et al. 2013).

\section{IN VIVO RELEVANCE: ROLE OF HISTONE VARIANTS AND HISTONE EXCHANGE IN ESTABLISHING PLASTICITY PROGRAMS}

Because of their distinct biochemical properties, histone variants, when incorporated into nucleosomes, confer specific features to the chromatin. From the above discussion, it is clear that modifying global chromatin properties through variations in histone composition is a key requirement for reprogramming. The examples discussed above illustrate that this is not only the case for in vivo reprogramming (Fig. 2A) but also in SCNT (Fig. 2B) and during transcription factor-mediated reprogramming to pluripotency (Fig. 2C). This implies that the histone chaperones and assembly factors themselves are expected to participate in establishing cellular plasticity.

After protamine removal, the male pronucleus contains an unusually high proportion of the H3.3 variant (TorresPadilla et al. 2006; Lin et al. 2014). Subsequently, during the following cell divisions H3.3 is "diluted" by the replication-dependent incorporation of canonical H3.1 and 
H3.2 mediated by CAF1. Thus, CAF1-dependent histone deposition onto the chromatin in the embryo and in ES cells is anticorrelated with the plasticity state of the cells. Developmentally, totipotent cells are predisposed to generate pluripotent cells. We postulate that the transition from totipotency to pluripotency is characterized by a switch from a H3.3-driven chromatin architecture toward a more H3.1/H3.2-enriched chromatin (Fig. 3). CAF-1 is therefore essential for this process.

In vivo, the epigenetic state of the male chromatin is reset into a totipotent state through histone and protamine removal, either during spermiogenesis or at fertilization, respectively. In analogy to this resetting event, recent work has shown that down-regulation of chromatin assembly activity in ES cells induces the formation of twocell-like cells, characterized by the acquisition of molecular features of totipotent cells (Ishiuchi et al. 2015). These findings suggest that a global reduction in nucleosomal occupancy promotes a cellular plasticity state that resembles that of the early embryo. Both together advocate for a very simple model whereby "erasing" information through removal of the basic unit of the chromatin itself promotes cellular plasticity (Fig. 3).

While these observations pertain histone $\mathrm{H} 3$, we expect that other histone variants might also contribute to main- tain a more accessible chromatin state. An open chromatin structure is presumably important to establish totipotency features including high chromatin mobility (Bošković et al. 2014), the absence of chromocenters (Ishiuchi et al. 2015), and activation of endogenous retroelements (Macfarlan et al. 2012). How these features trigger totipotency in the physiological sense, or whether they are rather a consequence of the totipotency state, is unknown. Furthermore, the possible stabilization of the totipotent state has not been addressed. This stabilization may involve specific histone (canonical or variant) posttranslational modifications, linker histone isoforms, and/ or the incorporation of other histone variants. Importantly, studying histone dynamics and replacement in the early embryo and in two-cell-like cells might answer these questions and help in understanding how global changes in chromatin composition regulate totipotency.

\section{CONCLUSION}

The transitions between totipotency and pluripotency are accompanied by drastic changes in chromatin signatures. In particular, the incorporation and eviction of histone variants from the chromatin are key actors in the

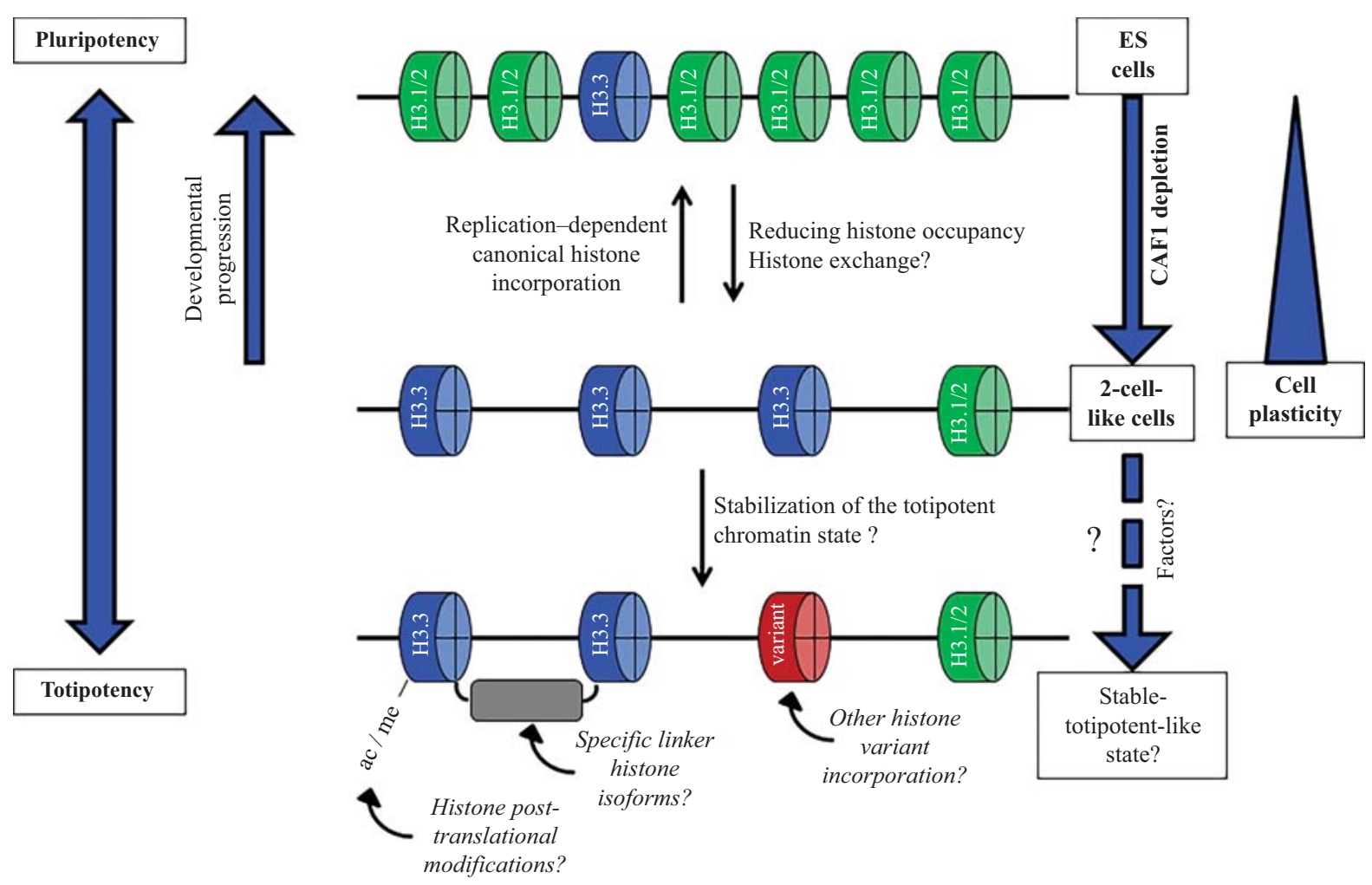

Figure 3. Role of histone variants in establishing totipotent chromatin. Totipotent chromatin is characterized by reduced histone occupancy and a high proportion of the H3.3 histone variant. During developmental progression, replication-dependent canonical histone incorporation in the chromatin is associated with a dilution of $\mathrm{H} 3.3$ histone variant and a decreased cellular plasticity toward pluripotency. In vitro, totipotent-like features are obtained by inhibiting replication-dependent histone incorporation. The resulting two-cell-like cells possess similar chromatin characteristics compared with the two-cell-stage embryo. Whether this totipotent chromatin state can be stabilized is unknown. Such stabilization may involve specific histone (canonical or variant) posttranslational modifications, specific linker histone isoforms, and/or the incorporation of other histone variants. ES, embryonic stem. 
establishment of plasticity programs. Although the role of H3.3 and its specific chaperones in totipotent versus pluripotent chromatin is starting to be understood, the interplay between histone variants during this process should be the subject of future investigations. How the specific combination of histone variants can trigger the establishment of a totipotent chromatin is unknown. Furthermore, we anticipate that the role of specific histone exchange factors is essential during the transitions of cell potency states and the acquisition of totipotency.

\section{ACKNOWLEDGMENTS}

We thank A. Burton for critical reading of the manuscript. Work in the M.-E.T.-P. laboratory is funded through EpiGeneSys NoE, ERC-Stg "NuclearPotency," EMBO Young Investigator Programme and the Schlumberger Foundation for Research and Education.

\section{REFERENCES}

Alami R, Fan Y, Pack S, Sonbuchner TM, Besse A, Lin Q, Greally JM, Skoultchi AI, Bouhassira EE. 2003. Mammalian linker-histone subtypes differentially affect gene expression in vivo. Proc Natl Acad Sci 100: 5920-5925.

Angelov D, Molla A, Perche PY, Hans F, Côté J, Khochbin S, Bouvet P, Dimitrov S. 2003. The histone variant macroH2A interferes with transcription factor binding and SWI/SNF nucleosome remodeling. Mol Cell 11: 1033-1041.

Apostolou E, Hochedlinger K. 2013. Chromatin dynamics during cellular reprogramming. Nature 502: 462-471.

Bao YH, Konesky K, Park YJ, Rosu S, Dyer PN, Rangasamy D, Tremethick DJ, Laybourn PJ, Luger K. 2004. Nucleosomes containing the histone variant H2A.Bbd organize only 118 base pairs of DNA. EMBO J 23: 3314-3324.

Barrero MJ, Sese B, Kuebler B, Bilic J, Boue S, Martí M, Izpisua Belmonte JC. 2013. Macrohistone variants preserve cell identity by preventing the gain of $\mathrm{H} 3 \mathrm{~K} 4 \mathrm{me} 2$ during reprogramming to pluripotency. Cell Rep 3: 1005-1011.

Bošković A, Bender A, Gall L, Ziegler-Birling C, Beaujean N, Torres-Padilla ME. 2012. Analysis of active chromatin modifications in early mammalian embryos reveals uncoupling of H2A.Z acetylation and H3K36 trimethylation from embryonic genome activation. Epigenetics 7: 747-757.

Bošković A, Eid A, Pontabry J, Ishiuchi T, Spiegelhalter C, Raghu Ram EV, Meshorer E, Torres-Padilla ME. 2014. Higher chromatin mobility supports totipotency and precedes pluripotency in vivo. Gene Dev 28: 1042-1047.

Brykczynska U, Hisano M, Erkek S, Ramos L, Oakeley EJ, Roloff TC, Beisel C, Schübeler D, Stadler MB, Peters AH. 2010. Repressive and active histone methylation mark distinct promoters in human and mouse spermatozoa. Nat Struct Mol Biol 17: 679-687.

Burton A, Torres-Padilla ME. 2014. Chromatin dynamics in the regulation of cell fate allocation during early embryogenesis. Nat Rev Mol Cell Bio 15: 722-734.

Celeste A, Petersen S, Romanienko PJ, Fernandez-Capetillo O, Chen HT, Sedelnikova OA, Reina-San-Martin B, Coppola V, Meffre E, Difilippantonio MJ, et al. 2002. Genomic instability in mice lacking histone H2AX. Science 296: 922-927.

Chadwick BP, Willard HF. 2001. A novel chromatin protein, distantly related to histone $\mathrm{H} 2 \mathrm{~A}$, is largely excluded from the inactive X chromosome. J Cell Biol 152: 375-384.

Chang CC, Ma Y, Jacobs S, Tian XC, Yang X, Rasmussen TP. 2005. A maternal store of macroH2A is removed from pronuclei prior to onset of somatic macroH2A expression in preimplantation embryos. Dev Biol 278: 367-380.
Chang CC, Gao S, Sung LY, Corry GN, Ma Y, Nagy ZP, Tian XC, Rasmussen TP. 2010. Rapid elimination of the histone variant macroH2A from somatic cell heterochromatin after nuclear transfer. Cell Reprogram 12: 43-53.

Chen P, Zhao J, Wang Y, Wang M, Long H, Liang D, Huang L, Wen Z, Li W, Li X, et al. 2013. H3.3 actively marks enhancers and primes gene transcription via opening higher-ordered chromatin. Gene Dev 27: 2109-2124.

Christophorou MA, Castelo-Branco G, Halley-Stott RP, Oliveira CS, Loos R, Radzisheuskaya A, Mowen KA, Bertone P, Silva JC, Zernicka-Goetz M, et al. 2014. Citrullination regulates pluripotency and histone H1 binding to chromatin. Nature 507: 104-108.

Clausell J, Happel N, Hale TK, Doenecke D, Beato M. 2009. Histone H1 subtypes differentially modulate chromatin condensation without preventing ATP-dependent remodeling by SWI/SNF or NURF. PLoS One 4: e0007243.

Costanzi C, Pehrson JR. 1998. Histone macroH2A1 is concentrated in the inactive $\mathrm{X}$ chromosome of female mammals. Nature 393: 599-601.

Doyen CM, An W, Angelov D, Bondarenko V, Mietton F, Studitsky VM, Hamiche A, Roeder RG, Bouvet P, Dimitrov S. 2006. Mechanism of polymerase II transcription repression by the histone variant macroH2A. Mol Cell Biol 26: 1156-1164.

Drané P, Ouararhni K, Depaux A, Shuaib M, Hamiche A. 2010. The death-associated protein DAXX is a novel histone chaperone involved in the replication-independent deposition of H3.3. Genes Dev 24: 1253-1265.

Eirín-López JM, Ishibashi T, Ausió J. 2008. H2A.Bbd: A quickly evolving hypervariable mammalian histone that destabilizes nucleosomes in an acetylation-independent way. FASEB J 22: $316-326$.

Eirín-López JM, González-Romero R, Dryhurst D, Ishibashi T, Ausió J. 2009. The evolutionary differentiation of two histone H2A.Z variants in chordates (H2A.Z-1 and H2A.Z-2) is mediated by a stepwise mutation process that affects three amino acid residues. BMC Evol Biol 9: 31.

Elsässer SJ, Noh KM, Diaz N, Allis CD, Banaszynski LA. 2015. Histone H3.3 is required for endogenous retroviral element silencing in embryonic stem cells. Nature 522: 240-244.

Faast R, Thonglairoam V, Schulz TC, Beall J, Wells JR, Taylor H, Matthaei K, Rathjen PD, Tremethick DJ, Lyons I. 2001. Histone variant H2A.Z is required for early mammalian development. Curr Biol 11: 1183-1187.

Fan Y, Sirotkin A, Russell RG, Ayala J, Skoultchi AI. 2001. Individual somatic H1 subtypes are dispensable for mouse development even in mice lacking the H1(0) replacement subtype. Mol Cell Biol 21: 7933-7943.

Fan JY, Rangasamy D, Luger K, Tremethick DJ. 2004. H2A.Z alters the nucleosome surface to promote HP1 $\alpha$-mediated chromatin fiber folding. Mol Cell 16: 655-661.

Fernandez-Capetillo O, Lee A, Nussenzweig M, Nussenzweig A. 2004. H2AX: The histone guardian of the genome. DNA Repair (Amst) 3: 959-967.

Gao S, Chung YG, Parseghian MH, King GJ, Adashi EY, Latham KE. 2004. Rapid H1 linker histone transitions following fertilization or somatic cell nuclear transfer: Evidence for a uniform developmental program in mice. Dev Biol 266: 62 75.

Gaspar-Maia A, Qadeer ZA, Hasson D, Ratnakumar K, Leu NA, Leroy G, Liu S, Costanzi C, Valle-Garcia D, Schaniel C, et al. 2013. MacroH2A histone variants act as a barrier upon reprogramming towards pluripotency. Nat Commun 4: 1565.

Gaume X, Monier K, Argoul F, Mongelard F, Bouvet P. 2011. In vivo study of the histone chaperone activity of nucleolin by FRAP. Biochem Res Int 2011: 187624.

Gautier T, Abbott DW, Molla A, Verdel A, Ausio J, Dimitrov S. 2004. Histone variant H2ABbd confers lower stability to the nucleosome. EMBO Rep 5: 715-720.

Goldberg AD, Banaszynski LA, Noh KM, Lewis PW, Elsaesser SJ, Stadler S, Dewell S, Law M, Guo X, Li X, et al. 2010. Distinct factors control histone variant H3.3 localization at specific genomic regions. Cell 140: 678-691. 
González F, Georgieva D, Vanoli F, Shi ZD, Stadtfeld M, Ludwig T, Jasin M, Huangfu D. 2013. Homologous recombination DNA repair genes play a critical role in reprogramming to a pluripotent state. Cell Rep 3: 651-660.

Govin J, Escoffier E, Rousseaux S, Kuhn L, Ferro M, Thévenon J, Catena R, Davidson I, Garin J, Khochbin S, et al. 2007. Pericentric heterochromatin reprogramming by new histone variants during mouse spermiogenesis. J Cell Biol 176: 283-294.

Gurdon JB, Melton DA. 2008. Nuclear reprogramming in cells. Science 322: 1811-1815.

Hajkova P, Jeffries SJ, Lee C, Miller N, Jackson SP, Surani MA. 2010. Genome-wide reprogramming in the mouse germ line entails the base excision repair pathway. Science 329: 78-82.

Hake SB, Allis CD. 2006. Histone H3 variants and their potential role in indexing mammalian genomes: The "H3 barcode hypothesis". Proc Natl Acad Sci 103: 6428-6435.

Hammoud SS, Nix DA, Zhang H, Purwar J, Carrell DT, Cairns BR. 2009. Distinctive chromatin in human sperm packages genes for embryo development. Nature 460: 473-478.

Happel N, Doenecke D. 2009. Histone H1 and its isoforms: Contribution to chromatin structure and function. Gene 431: $1-12$.

Hayakawa K, Ohgane J, Tanaka S, Yagi S, Shiota K. 2012. Oocyte-specific linker histone H1 foo is an epigenomic modulator that decondenses chromatin and impairs pluripotency. Epigenetics 7: 1029-1036.

Hödl M, Basler K. 2009. Transcription in the absence of histone H3.3. Curr Biol 19: 1221-1226.

Inoue A, Zhang Y. 2014. Nucleosome assembly is required for nuclear pore complex assembly in mouse zygotes. Nat Struct Mol Biol 21: 609-616.

Ishiuchi T, Enriquez-Gasca R, Mizutani E, Bošković A, ZieglerBirling C, Rodriguez-Terrones D, Wakayama T, Vaquerizas JM, Torres-Padilla ME. 2015. Early embryonic-like cells are induced by downregulating replication-dependent chromatin assembly. Nat Struct Mol Biol 22: 662-671.

Jang CW, Shibata Y, Starmer J, Yee D, Magnuson T. 2015. Histone H3.3 maintains genome integrity during mammalian development. Genes Dev 29: 1377-1392.

Jin C, Felsenfeld G. 2007. Nucleosome stability mediated by histone variants H3.3 and H2A.Z. Genes Dev 21: 1519-1529.

Jin C, Zang C, Wei G, Cui K, Peng W, Zhao K, Felsenfeld G. 2009. H3.3/H2A.Z double variant-containing nucleosomes mark 'nucleosome-free regions' of active promoters and other regulatory regions. Nat Genet 41: 941-945.

Jullien J, Astrand C, Halley-Stott RP, Garrett N, Gurdon JB. 2010. Characterization of somatic cell nuclear reprogramming by oocytes in which a linker histone is required for pluripotency gene reactivation. Proc Natl Acad Sci 107: $5483-5488$.

Jullien J, Astrand C, Szenker E, Garrett N, Almouzni G, Gurdon JB. 2012. HIRA dependent H3.3 deposition is required for transcriptional reprogramming following nuclear transfer to Xenopus oocytes. Epigenetics Chromatin 5: 17.

Kamieniarz K, Izzo A, Dundr M, Tropberger P, Ozretic L, Kirfel J, Scheer E, Tropel P, Wisniewski JR, Tora L, et al. 2012. A dual role of linker histone H1.4 Lys 34 acetylation in transcriptional activation. Genes Dev 26: 797-802.

Konev AY, Tribus M, Park SY, Podhraski V, Lim CY, Emelyanov AV, Vershilova E, Pirrotta V, Kadonaga JT, Lusser A, et al. 2007. CHD1 motor protein is required for deposition of histone variant H3.3 into chromatin in vivo. Science 317: 1087-1090.

Lewis PW, Elsaesser SJ, Noh KM, Stadler SC, Allis CD. 2010. Daxx is an H3.3-specific histone chaperone and cooperates with ATRX in replication-independent chromatin assembly at telomeres. Proc Natl Acad Sci 107: 14075-14080.

Lin QC, Sirotkin A, Skoultchi AI. 2000. Normal spermatogenesis in mice lacking the testis-specific linker histone H1t. Mol Cell Biol 20: 2122-2128.

Lin CJ, Koh FM, Wong P, Conti M, Ramalho-Santos M. 2014. Hira-mediated H3.3 incorporation is required for DNA replication and ribosomal RNA transcription in the mouse zygote. Dev Cell 30: 268-279.
Luk E, Vu ND, Patteson K, Mizuguchi G, Wu WH, Ranjan A, Backus J, Sen S, Lewis M, Bai Y, et al. 2007. Chz1, a nuclear chaperone for histone H2AZ. Mol Cell 25: 357-368.

Macfarlan TS, Gifford WD, Driscoll S, Lettieri K, Rowe HM, Bonanomi D, Firth A, Singer O, Trono D, Pfaff SL. 2012. Embryonic stem cell potency fluctuates with endogenous retrovirus activity. Nature 487: 57-63.

Mao Z, Pan L, Wang W, Sun J, Shan S, Dong Q, Liang X, Dai LC, Ding X, Chen S, et al. 2014. Anp32e, a higher eukaryotic histone chaperone directs preferential recognition for H2A.Z. Cell Res 24: 389-399.

Marion RM, Strati K, Li H, Murga M, Blanco R, Ortega S, Fernandez-Capetillo O, Serrano M, Blasco MA. 2009. A p53-mediated DNA damage response limits reprogramming to ensure iPS cell genomic integrity. Nature 460: 1149-1153.

McBryant SJ, Lu X, Hansen JC. 2010. Multifunctionality of the linker histones: An emerging role for protein-protein interactions. Cell Res 20: 519-528.

Minajigi A, Froberg JE, Wei C, Sunwoo H, Kesner B, Colognori D, Lessing D, Payer B, Boukhali M, Haas W, et al. 2015. A comprehensive Xist interactome reveals cohesin repulsion and an RNA-directed chromosome conformation. Science 349: 282.

Mizuguchi G, Shen X, Landry J, Wu WH, Sen S, Wu C. 2004. ATP-driven exchange of histone $\mathrm{H} 2 \mathrm{AZ}$ variant catalyzed by SWR1 chromatin remodeling complex. Science 303: $343-$ 348.

Montellier E, Boussouar F, Rousseaux S, Zhang K, Buchou T, Fenaille F, Shiota H, Debernardi A, Héry P, Curtet S, et al. 2013. Chromatin-to-nucleoprotamine transition is controlled by the histone H2B variant TH2B. Genes Dev 27: 1680-1692.

Nashun B, Yukawa M, Liu H, Akiyama T, Aoki F. 2010. Changes in the nuclear deposition of histone H2A variants during pre-implantation development in mice. Development 137: 3785-3794.

Nashun B, Akiyama T, Suzuki MG, Aoki F. 2011. Dramatic replacement of histone variants during genome remodeling in nuclear-transferred embryos. Epigenetics 6: 1489-1497.

Noh KM, Maze I, Zhao D, Xiang B, Wenderski W, Lewis PW, Shen L, Li HT, Allis CD. 2015. ATRX tolerates activity-dependent histone $\mathrm{H} 3$ methyl/phos switching to maintain repetitive element silencing in neurons. Proc Natl Acad Sci 112: 6820-6827.

Obri A, Ouararhni K, Papin C, Diebold ML, Padmanabhan K, Marek M, Stoll I, Roy L, Reilly PT, Mak TW, et al. 2014. ANP32E is a histone chaperone that removes H2A.Z from chromatin. Nature 505: 648-653.

Pasque V, Gillich A, Garrett N, Gurdon JB. 2011. Histone variant macroH2A confers resistance to nuclear reprogramming. EMBO J 30: 2373-2387.

Pehrson JR, Fried VA. 1992. Macroh2a, a core histone containing a large nonhistone region. Science 257: 1398-1400.

Pehrson JR, Changolkar LN, Costanzi C, Leu NA. 2014. Mice without macroH2A histone variants. Mol Cell Biol 34: $4523-$ 4533.

Rabini S, Franke K, Saftig P, Bode C, Doenecke D, Drabent B. 2000. Spermatogenesis in mice is not affected by histone H1.1 deficiency. Exp Cell Res 255: 114-124.

Rangasamy D, Berven L, Ridgway P, Tremethick DJ. 2003. Pericentric heterochromatin becomes enriched with $\mathrm{H} 2 \mathrm{~A}$. $\mathrm{Z}$ during early mammalian development. EMBO J 22: 1599-1607.

Rogakou EP, Pilch DR, Orr AH, Ivanova VS, Bonner WM. 1998. DNA double-stranded breaks induce histone H2AX phosphorylation on serine 139. J Biol Chem 273: 5858-5868.

Sakai A, Schwartz BE, Goldstein S, Ahmad K. 2009. Transcriptional and developmental functions of the H3.3 histone variant in Drosophila. Curr Biol 19: 1816-1820.

Santenard A, Ziegler-Birling C, Koch M, Tora L, Bannister AJ, Torres-Padilla ME. 2010. Heterochromatin formation in the mouse embryo requires critical residues of the histone variant H3.3. Nat Cell Biol 12: 853-862.

Schenk R, Jenke A, Zilbauer M, Wirth S, Postberg J. 2011. H3.5 is a novel hominid-specific histone $\mathrm{H} 3$ variant that is specif- 
ically expressed in the seminiferous tubules of human testes. Chromosoma 120: 275-285.

Schwartz BE, Ahmad K. 2005. Transcriptional activation triggers deposition and removal of the histone variant H3.3. Gene Dev 19: 804-814.

Shinagawa T, Takagi T, Tsukamoto D, Tomaru C, Huynh LM, Sivaraman P, Kumarevel T, Inoue K, Nakato R, Katou Y, et al. 2014. Histone variants enriched in oocytes enhance reprogramming to induced pluripotent stem cells. Cell Stem Cell 14: $217-227$.

Shires A, Carpenter MP, Chalkley R. 1976. Cysteine-containing $\mathrm{H} 2 \mathrm{~b}$-like histone found in mature mammalian testis. $J$ Biol Chem 251: 4155-4158.

Sirotkin AM, Edelmann W, Cheng G, Klein-Szanto A, Kucherlapati R, Skoultchi AI. 1995. Mice develop normally without the $\mathrm{H}^{0}$ linker histone. Proc Natl Acad Sci 92: 6434-6438.

Soboleva TA, Nekrasov M, Pahwa A, Williams R, Huttley GA, Tremethick DJ. 2012. A unique H2A histone variant occupies the transcriptional start site of active genes. Nat Struct Mol Biol 19: 25-30

Tagami H, Ray-Gallet D, Almouzni G, Nakatani Y. 2004. Histone H3.1 and H3.3 complexes mediate nucleosome assembly pathways dependent or independent of DNA synthesis. Cell 116: $51-61$.

Takahashi K, Yamanaka S. 2006. Induction of pluripotent stem cells from mouse embryonic and adult fibroblast cultures by defined factors. Cell 126: 663-676.

Tanaka M, Hennebold JD, Macfarlane J, Adashi EY. 2001. A mammalian oocyte-specific linker histone gene H1oo: Homology with the genes for the oocyte-specific cleavage stage histone (cs-H1) of sea urchin and the B4/H1M histone of the frog. Development 128: 655-664.

Teranishi T, Tanaka M, Kimoto S, Ono Y, Miyakoshi K, Kono T, Yoshimura Y. 2004. Rapid replacement of somatic linker histones with the oocyte-specific linker histone H1 foo in nuclear transfer. Dev Biol 266: 76-86.

Terme JM, Sesé B, Millán-Ariño L, Mayor R, Izpisúa Belmonte JC, Barrero MJ, Jordan A. 2011. Histone H1 variants are differentially expressed and incorporated into chromatin during differentiation and reprogramming to pluripotency. $\mathrm{J} \mathrm{Biol}$ Chem 286: 35347-35357.

Tolstorukov MY, Goldman JA, Gilbert C, Ogryzko V, Kingston RE, Park PJ. 2012. Histone variant H2A.Bbd is associated with active transcription and mRNA processing in human cells. Mol Cell 47: 596-607.

Torres-Padilla ME, Bannister AJ, Hurd PJ, Kouzarides T, Zernicka-Goetz M. 2006. Dynamic distribution of the replacement histone variant $\mathrm{H} 3.3$ in the mouse oocyte and preimplantation embryos. Int J Dev Biol 50: 455-461.

Trostle-weige PK, Meistrich ML, Brock WA, Nishioka K, Bremer JW. 1982. Isolation and characterization of Th2A, a germ cell-specific variant of histone $2 \mathrm{~A}$ in rat testis. $J$ Biol Chem 257: 5560-5567.

Turinetto V, Giachino C. 2015. Multiple facets of histone variant H2AX: A DNA double-strand-break marker with several biological functions. Nucleic Acids Res 43: 2489-2498. van der Heijden GW, Dieker JW, Derijck AA, Muller S, Berden $\mathrm{JH}$, Braat DD, van der Vlag J, de Boer P. 2005. Asymmetry in Histone $\mathrm{H} 3$ variants and lysine methylation between paternal and maternal chromatin of the early mouse zygote. Mech Develop 122: 1008-1022.

van der Heijden GW, Derijck AA, Pósfai E, Giele M, Pelczar P, Ramos L, Wansink DG, van der Vlag J, Peters AH, de Boer P. 2007. Chromosome-wide nucleosome replacement and H3.3 incorporation during mammalian meiotic sex chromosome inactivation. Nat Genet 39: 251-258.

Wang J, Qiao M, He Q, Shi R, Loh SJ, Stanton LW, Wu M. 2015. Pluripotency activity of nanog requires biochemical stabilization by variant histone protein H2A.Z. Stem Cells 33: $2126-$ 2134.

Wellman SE, Casano PJ, Pilch DR, Marzluff WF, Sittman DB. 1987. Characterization of mouse H3.3-like histone genes. Gene 59: 29-39.

Wen D, Banaszynski LA, Rosenwaks Z, Allis CD, Rafii S. 2014. H3.3 replacement facilitates epigenetic reprogramming of donor nuclei in somatic cell nuclear transfer embryos. Nucleus 5: 369-375.

Wiedemann SM, Mildner SN, Bönisch C, Israel L, Maiser A, Matheisl S, Straub T, Merkl R, Leonhardt H, Kremmer E, et al. 2010. Identification and characterization of two novel primate-specific histone H3 variants, H3.X and H3.Y. J Cell Biol 190: 777-791.

Witt O, Albig W, Doenecke D. 1996. Testis-specific expression of a novel human $\mathrm{H} 3$ histone gene. Exp Cell Res 229: $301-$ 306.

Wong LH, Ren H, Williams E, McGhie J, Ahn S, Sim M, Tam A, Earle E, Anderson MA, Mann J, et al. 2009. Histone H3.3 incorporation provides a unique and functionally essential telomeric chromatin in embryonic stem cells. Genome Res 19: $404-414$.

Wong LH, McGhie JD, Sim M, Anderson MA, Ahn S, Hannan RD, George AJ, Morgan KA, Mann JR, Choo KH. 2010. ATRX interacts with H3.3 in maintaining telomere structural integrity in pluripotent embryonic stem cells. Genome Res 20: $351-360$.

Wossidlo M, Arand J, Sebastiano V, Lepikhov K, Boiani M, Reinhardt R, Schöler H, Walter J. 2010. Dynamic link of DNA demethylation, DNA strand breaks and repair in mouse zygotes. EMBO J 29: 1877-1888.

Wu T, Liu Y, Wen D, Tseng Z, Tahmasian M, Zhong M, Rafii S, Stadtfeld M, Hochedlinger K, Xiao A. 2014. Histone variant H2A.X deposition pattern serves as a functional epigenetic mark for distinguishing the developmental potentials of iPSCs. Cell Stem Cell 15: 281-294.

Zhou J, Fan JY, Rangasamy D, Tremethick DJ. 2007. The nucleosome surface regulates chromatin compaction and couples it with transcriptional repression. Nat Struct Mol Biol 14: 1070-1076.

Ziegler-Birling C, Helmrich A, Tora L, Torres-Padilla ME. 2009. Distribution of p53 binding protein 1 (53BP1) and phosphorylated H2A.X during mouse preimplantation development in the absence of DNA damage. Int J Dev Biol 53: 1003-1011. 


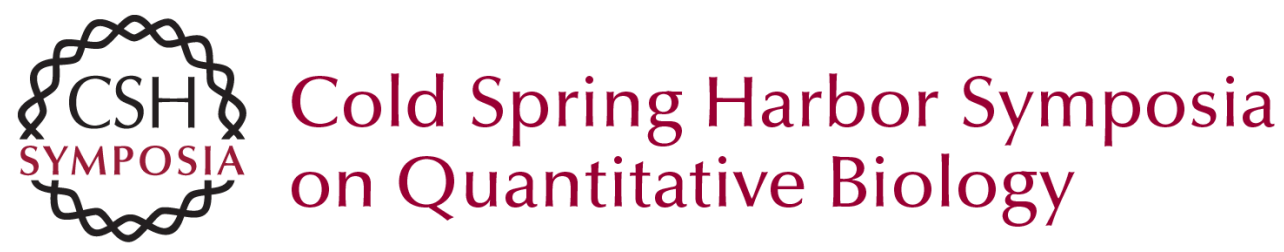

\section{Regulation of Reprogramming and Cellular Plasticity through Histone Exchange and Histone Variant Incorporation}

Xavier Gaume and Maria-Elena Torres-Padilla

Cold Spring Harb Symp Quant Biol 2015 80: 165-175 originally published online November 18, 2015

Access the most recent version at doi:10.1101/sqb.2015.80.027458

References This article cites 100 articles, 40 of which can be accessed free at:

http://symposium.cshlp.org/content/80/165.full.html\#ref-list-1

License

Email Alerting Receive free email alerts when new articles cite this article - sign up in Service the box at the top right corner of the article or click here. 